\title{
Concordance of the management of chronic gout in a UK primary-care population with the EULAR gout recommendations
}

\author{
Edward Roddy, Weiya Zhang, Michael Doherty
}

See linked editorial, p1269

Ann Rheum Dis 2007;66:1311-1315. doi: 10.1136/ard.2007.070755

See end of article for authors' affiliations

Correspondence to:

Dr Edward Roddy, Clinical Research Fellow \&

Consultant Rheumatologist, Primary Care

Musculoskeletal Research

Centre, Keele University,

Staffordshire, ST5 5BG;

e.roddy@cphc.keele.ac.uk

Accepted 28 April 2007

Published Online First

15 May 2007
Objectives: To assess concordance of the management of chronic gout in UK primary care with the European League Against Rheumatism (EULAR) gout recommendations.

Methods: A postal questionnaire was sent to all adults aged $>30$ years registered with two general practices. Patients with possible gout attended for clinical assessment, at which the diagnosis was verified clinically. Aspects of chronic gout management, including provision of lifestyle modification advice, use of uratelowering therapies (ULT) including dose titration to serum urate (SUA) level, prophylaxis against acute attacks, and diuretic cessation were assessed in accordance with the EULAR recommendations.

Results: Of $4249(32 \%)$ completed questionnaires returned, 488 reported gout or acute attacks and were invited for clinical assessment. Of 359 attendees, 164 clinically confirmed cases of gout were identified. Advice regarding alcohol consumption was recalled by $59(41 \%)$, weight loss by $36(25 \%)$ and diet by 42 (29\%). Allopurinol was the only ULT used and was taken by 44 (30\%); 31 (70\%) were taking $300 \mathrm{mg}$ daily. Mean SUA was lower in allopurinol users than non-users $(318$ vs $434 \mu \mathrm{mol} / \mathrm{l})$ and was less often $>360 \mu \mathrm{mol} / \mathrm{l}$ in allopurinol users (23\% vs $75 \%$ ). Eight patients had recently commenced allopurinol; two of these also were taking prophylactic colchicine or non-steroidal anti-inflammatory drugs. Of 25 patients with diuretic-induced gout, 16 (64\%) were still taking a diuretic.

Conclusion: Treatment of chronic gout is often suboptimal and poorly concordant with EULAR recommendations. Lifestyle advice is infrequently offered, and allopurinol is restricted to a minority. Persistent hyperuricaemia was often seen in allopurinol non-users, but was also in allopurinol users, suggesting that doses $>300 \mathrm{mg}$ are often necessary.
7 out is one of the most prevalent causes of inflammatory arthritis, affecting $1-2 \%$ of adults in the UK. ${ }^{1}$ It is the most common cause of inflammatory arthritis in men, with a male:female ratio of $3-5: 1 .^{1-3}$ In contrast to the other common inflammatory arthritides, gout is largely managed in primary care in the UK. Since the advent of effective uratelowering therapies (ULT) such as allopurinol and uricosuric agents, gout is also considered to be one of the few curable arthritides. However, clinical experience suggests that gout is frequently misdiagnosed and/or mismanaged. In 2006, the European League Against Rheumatism (EULAR) Gout Task Force produced recommendations for the diagnosis and management of gout. ${ }^{4}$ These recommendations emphasise that the optimum long-term management of gout should include patient education, modification of adverse lifestyle factors such as diet, body weight and alcohol, and titration of the dose of ULT with the aim of reducing the serum urate (SUA) level to below the physiological saturation threshold of urate in the serum - that is, approximately $380 \mu \mathrm{mol} / \mathrm{l}(6.4 \mathrm{mg} /$ $\mathrm{dL})$. Recent studies have suggested that reduction of SUA to $<360 \mu \mathrm{mol} / \mathrm{l}(6 \mathrm{mg} / \mathrm{dL})$ decreases the frequency of acute attacks and reduces crystal load. ${ }^{67}$ However, little is known about disease control and quality of treatment in those patients managed outside rheumatology clinics, who make up the majority of patients with gout.

We undertook a survey of the management of chronic gout in a primary care-based population, including assessment of the provision of lifestyle modification advice and written educational material, use of ULT (including dosage) and evaluation of the degree of control obtained, to examine concordance with the EULAR recommendations for the management of gout.

\section{METHODS}

The study was undertaken in two general practices in Nottingham, UK, nested within a case-control study. It consisted of two phases: (1) a postal questionnaire survey and (2) a face-to-face clinical assessment. The study was approved by the Nottingham Local Research Ethics Committee 2, and written consent to participate was obtained from participants who attended for clinical assessment.

\section{The 2006 EULAR recommendations for the management of gout}

The 2006 EULAR recommendations for the management of gout consist of 12 propositions relating to key clinical aspects in the management of gout. ${ }^{5}$ One proposition describes general principles for the optimum treatment of gout (proposition 1), one considers associated cardiovascular risk factors and comorbidity (3), and three propositions (4-6) relate to the treatment of the acute attack of gout. The remaining seven propositions relate to the management of chronic gout and were thought suitable for assessment in this study.

\section{Postal questionnaire}

Each practice drew up a list of all registered adults $>30$ years old, excluding those with a history of major psychiatric disease, dementia or recently diagnosed malignancy. A questionnaire was then posted to all listed individuals with a pre-paid

Abbreviations: ARA, American Rheumatism Association; EULAR, European League Against Rheumatism; GFR, glomerular filtration rate; NSAID, non-steroidal anti-inflammatory drug; SUA, serum urate; ULT, urate-lowering therapies 
envelope included for their reply. Possible cases of gout were identified by two questions:

- 1) Have you ever been diagnosed with or suffered from gout?

- 2) Have you ever suffered from an acute attack of arthritis that was severely painful, was associated with a red and swollen joint, came on suddenly reaching its peak severity within 24 hours and then went away completely within 3 weeks? (That is, a typical attack of crystal synovitis.)

Current and previous use of allopurinol and diuretics were requested, in addition to recording of standard demographic information, weight, height and current medication use.

After the first round of posting, in order to enhance a poor response rate, an abbreviated questionnaire was posted out to non-respondents in one practice.

\section{Clinical assessment}

All patients indicating a previous diagnosis of gout or a history of acute self-limiting attacks of painful, red and swollen joints in their questionnaire (ie potential cases), were invited to attend a clinical assessment. At this visit, the patient's joint problems were reviewed by a doctor with special training in gout (ER) and the most likely diagnosis was made on clinical grounds. The diagnosis of gout was further assessed according to the American Rheumatism Association (ARA) preliminary criteria for the acute arthritis of primary gout. ${ }^{8}$ Recall of the number of attacks in the past year and ever experienced was documented.

Subjects were asked whether, relating specifically to gout, they had ever been advised by a healthcare professional to restrict their alcohol intake, lose weight/avoid gaining weight, or avoid red meat or seafood. They were asked if they had ever been provided with written information about gout.

Participants were examined for presence of tophi. These were classified as definite (asymmetrical white/yellow swellings appearing when the overlying skin was pulled taut) or possible (asymmetrical nodular swellings without white/yellow discolouration). Blood was taken for measurement of SUA and creatinine.

\section{Analysis}

Possible cases of secondary gout were identified by use of diuretics before onset of gout and a calculated glomerular filtration rate (GFR) of $<60 \mathrm{ml} / \mathrm{min}$. Patients who had consulted a doctor about gout were divided into two groups according to current ULT (specifically allopurinol) use. Age, gender and disease duration of current allopurinol users were compared with non-users. Allopurinol dosage, disease duration prior to commencing allopurinol and length of treatment were also assessed in the group currently taking allopurinol. As a measure of adherence, patients taking allopurinol were asked to estimate, as a percentage, how often they took allopurinol as prescribed. The degree of control of current allopurinol users and non-users were compared by mean SUA, proportion of patients with a SUA $>360 \mu \mathrm{mol} / \mathrm{l}$ and the number of attacks experienced in the previous 12 months.

\section{Statistical analysis}

Comparison was made between normally distributed continuous variables using the independent samples $t$ test and nonnormally distributed variables using the Mann-Whitney U test. Categorical variables were compared using the $\chi^{2}$ test. The odds ratio (OR) between SUA ( $>$ or $<360 \mu \mathrm{mol} / \mathrm{l}$ ) and whether or not an attack of gout had occurred in the preceding year was calculated. The relationship between SUA and allopurinol dosing was assessed by a one-way analysis of variance for linearity. Analyses were performed using SPSS software V.11.0 (SPSS Inc., Chicago, IL, USA).

\section{RESULTS}

Questionnaires were posted to 13684 people, from which 4249 completed responses were received. In total, 294 were notified as being sent to the wrong address. Hence, the adjusted response rate was $32 \%$ (fig 1 ).

In all, 488 respondents reported acute attacks of self-limiting synovitis and/or a diagnosis of gout; 264 had been given a diagnosis of gout. A total of $359(75 \%)$ attended the clinical assessment. Of these, 175 reported being diagnosed with gout by a doctor; this was thought to be the correct diagnosis in 145 $(83 \%)$. A further 19 patients were thought to have gout but had

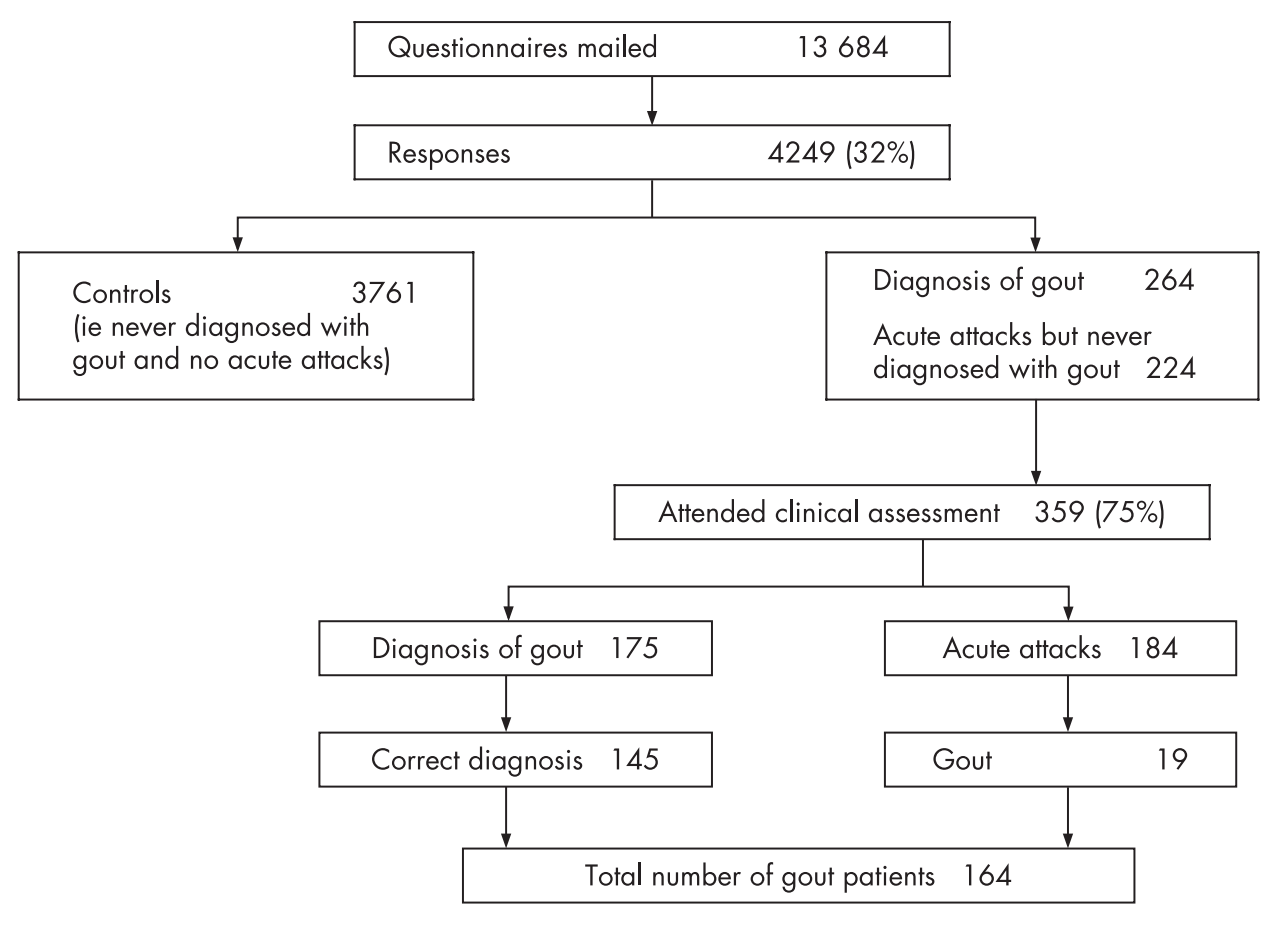

Figure 1 Flow of patients through the study. 
Table 1 Characteristics of patients with gout

\begin{tabular}{|c|c|}
\hline Characteristic & $\begin{array}{l}\text { Gout cases } \\
(n=164)\end{array}$ \\
\hline Age (years), mean (SD) & $63.4(11.2)$ \\
\hline Male gender, $\mathrm{n}(\%)$ & $133(81)$ \\
\hline $\mathrm{BMI}\left(\mathrm{kg} / \mathrm{m}^{2}\right)$, mean (SD) & $27.8(4.0)$ \\
\hline Age at first attack (years), mean (SD) & 50.5 (13.7) \\
\hline Disease duration (years), median (range) & $10(0-50)$ \\
\hline \multicolumn{2}{|l|}{ Tophus, n (\%) } \\
\hline Total & $10(6)$ \\
\hline Definite & 5 (3) \\
\hline Possible & $5(3)$ \\
\hline Fulfilled ARA preliminary criteria for primary gout (\%) & $150(92 \%)$ \\
\hline Serum urate $(\mu \mathrm{mol} / \mathrm{l})$, mean $(\mathrm{SD})^{*}$ & $399(107)$ \\
\hline Serum creatinine $(\mu \mathrm{mol} / \mathrm{l})$, median (range)t & $94(63$ to 180$)$ \\
\hline GFR ( $\mathrm{ml} / \mathrm{min})$, median (range) & 78 (19 to 170$)$ \\
\hline GFR $(<60 \mathrm{ml} / \mathrm{min}), \mathrm{n}(\%)$ & $39(24 \%)$ \\
\hline \multicolumn{2}{|l|}{ Medication use, $\mathrm{n}(\%)$} \\
\hline Taken diuretics prior to first attack & $25(15)$ \\
\hline Low-dose aspirin & $51(31)$ \\
\hline Allopurinol & $44(30)$ \\
\hline Uricosuric agents & 0 \\
\hline Possible secondary gout, $\mathrm{n}(\%) \ddagger$ & $50(30)$ \\
\hline
\end{tabular}

ARA, American Rheumatism Association; BMI, body mass index; GFR, glomerular filtration rate.

${ }^{*}$ Normal range $100-400 \mu \mathrm{mol} / \mathrm{l}$; tnormal range $60-120 \mu \mathrm{mol} / \mathrm{l}$; ‡GFR<60 $\mathrm{ml} / \mathrm{min}$ or taking diuretics prior to first altack.

never consulted a doctor. Therefore, 164 patients were identified as having gout, 19 (12\%) of whom had never consulted a doctor. Characteristics of the 164 patients with gout are shown in table 1 .

\section{Fulfilment of EULAR recommendations}

Proposition 2: patient education and appropriate lifestyle advice regarding weight loss if obese, diet, and reduced alcohol (especially beer) are core aspects of management

Recall of lifestyle modification advice and receipt of written educational material concerning gout by the 145 subjects who had consulted a doctor about gout is shown in figure 2 .

Proposition 7: ULT is indicated in patients with recurrent acute attacks, arthropathy, tophi, or radiographic changes of gout

In total, $44(30 \%)$ subjects were currently taking allopurinol. No subjects were taking uricosuric agents. There were 114 subjects who had experienced $>1$ acute attack of gout; $37(33 \%)$ of these were currently taking allopurinol and a further $18(16 \%)$ had taken allopurinol in the past. Of 10 patients with clinically evident tophi, $2(20 \%)$ were currently taking allopurinol, and an additional $4(40 \%)$ had taken allopurinol in the past. Chronic arthropathy and radiographic change were not assessed.

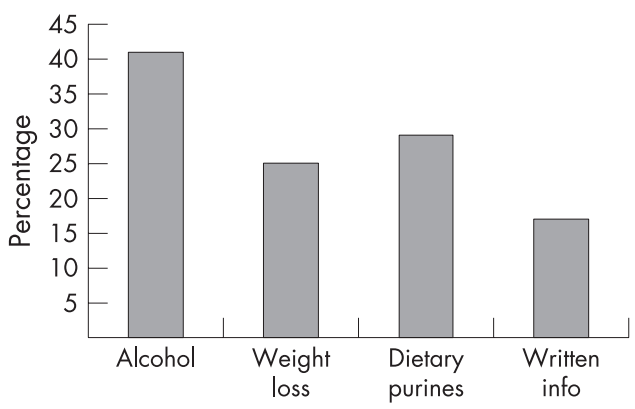

Figure 2 Recall of lifestyle-modification advice and provision of written educational material.
Proposition 8: the therapeutic goal of ULT is to promote crystal dissolution and prevent crystal formation; this is achieved by maintaining the serum uric acid below the saturation point for monosodium urate $(\leqslant 360 \mu \mathrm{mol} / \mathrm{l})$

Comparisons between current allopurinol users and non-users are shown in table 2. Mean SUA was significantly lower in current allopurinol users than non-users and the SUA was less often $>360 \mu \mathrm{mol} / \mathrm{l}$ in current allopurinol users than non-users. Fewer current allopurinol users had experienced an acute attack of gout in the preceding 12 months than had non-users. There was a significant association between SUA and acute attacks in the past year (OR $=2.68,95 \%$ CI 1.41 to 5.09).

Proposition 9: allopurinol is an appropriate long-term urate-lowering drug; it should be started at a low dose (eg, $100 \mathrm{mg}$ daily) and increased by $100 \mathrm{mg}$ every 2-4 weeks if required. The dose must be adjusted in patients with renal impairment; if allopurinol toxicity occurs, options include other xanthine oxidase inhibitors, a uricosuric agent or allopurinol desensitisation (the latter only in cases of mild rash) In total, $31(70 \%)$ current allopurinol users were taking a dose of $300 \mathrm{mg}$ daily. Five (11\%) patients were taking $100 \mathrm{mg}$ daily, whereas only four were taking a dose $>300 \mathrm{mg}$ daily. There was a significant inverse relationship between allopurinol dose and SUA on one-way analysis of variance for linearity (fig 3; $\mathrm{R}=-0.40, \mathrm{p}<0.01$ ).

Of 101 patients who were not currently taking allopurinol, 21 had taken allopurinol in the past but had since discontinued. Reasons for discontinuation are shown in table 3.

Proposition 10: uricosuric agents such as probenecid and sulfinpyrazone can be used as an alternative to allopurinol in patients with normal renal function but are relatively contraindicated in patients with urolithiasis; benzbromarone can be used in patients with mild to moderate renal insufficiency on a namedpatient basis but carries a small risk of hepatotoxicity No patients were taking uricosuric agents.

Proposition 11: prophylaxis against acute attacks during the first months of ULT can be achieved by colchicine (0.5-1 mg daily) and/or a non-steroidal anti-inflammatory drug (with gastroprotection if indicated)

Eight patients had commenced allopurinol within the preceding 6-month period. Two (25\%) of these were taking prophylactic colchicine and/or a non-steroidal anti-inflammatory drug (NSAID).

Proposition 12: when gout is associated with diuretic therapy, stop the diuretic if possible; for hypertension and hyperlipidaemia consider use of losartan and fenofibrate, respectively (both have modest uricosuric effects)

In total, 25 patients reported taking a diuretic at the time of the first acute attack of gout or had taken a diuretic before gout onset. The diuretic agent had not been discontinued in 16 (64\%).

\section{DISCUSSION}

In a primary care-derived population, the treatment of chronic gout appears to be suboptimal and poorly concordant with recent EULAR recommendations for the management of gout. ${ }^{5}$ Lifestyle-modification advice is infrequently offered and 
Table 2 Use of allopurinol in 145 patients who had consulted a doctor about gout

\begin{tabular}{lccc}
\hline & \multicolumn{2}{l}{ Current allopurinol use } & \\
\cline { 2 - 3 } \cline { 2 - 3 } & Yes & No & p Value \\
\hline $\mathrm{n}(\%)$ & $44(30 \%)$ & $101(70 \%)$ & - \\
Age (years), median (range) & $65(42$ to 79$)$ & $66(24$ to 83$)$ & 0.670 \\
Male gender, $\mathrm{n}(\%)$ & $42(96 \%)$ & $79(78 \%)$ & 0.010 \\
Disease duration (years), median (range) & $16(0$ to 44$)$ & $9(0$ to 50$)$ & 0.011 \\
Delay to starting allopurinol (months), median (range) & $48(0$ to 312$)$ & - & - \\
Length of treatment (months), median (range) & $120(1$ to 480$)$ & - & - \\
Self-reported adherence $<90 \%$ & $7(15 \%)$ & - & - \\
Serum urate $(\mu \mathrm{mol} / \mathrm{l})$, mean (SD) & $318(66)$ & $434(101)$ & $<0.001$ \\
Serum urate $>360$ mol//, $\mathrm{n}(\%)$ & $10(23 \%)$ & $75(75 \%)$ & $<0.001$ \\
At least 1 attack in preceding year & $14(32 \%)$ & $57(57 \%)$ & 0.005 \\
\hline
\end{tabular}

allopurinol is only prescribed for a minority of patients despite ongoing hyperuricaemia and recurrent acute attacks in allopurinol non-users. Although other UK studies ${ }^{2}{ }^{9}$ have identified higher rates of allopurinol use in primary care, our finding of $30 \%$ is consistent with the findings of a recent large study undertaken in the General Practice Research Database. ${ }^{1}$ Uricosuric use is commonly limited to secondary care in the UK.

Even in allopurinol users, disease control is often suboptimal. Almost one-third of allopurinol users had experienced acute attacks of gout in the preceding year despite a median duration of therapy of 10 years. Recent evidence suggests that the aim of ULT should be to lower SUA to $<360 \mu \mathrm{mol} / ;^{67}$ yet $23 \%$ of allopurinol users had not attained this goal. Our finding that $300 \mathrm{mg}$ is the most commonly prescribed dose of allopurinol is consistent with a number of studies from the UK and abroad, in both primary and secondary care. ${ }^{29-12}$ The maximum dose of allopurinol in the UK is $900 \mathrm{mg}$, and the suboptimum degree of control identified suggests that higher doses are warranted. Poor adherence is often cited as an important reason why treatment of gout may fail and, although self-reported adherence was reasonable in our survey, studies undertaken in prescribing databases suggest adherence to ULT to be low. ${ }^{13}{ }^{14}$ Another commonly stated barrier to successful use of allopurinol is hypersensitivity. This is thought to be a particular problem in the elderly and in those with impaired renal function. ${ }^{15}{ }^{16}$ However, none of the 65 patients who had ever taken allopurinol in this study had experienced a severe hypersensitivity reaction and only one patient had experienced a milder allergic reaction necessitating discontinuation of the drug. It is likely that hypersensitivity is a less significant problem in the spectrum of gout patients seen in primary care compared with hospital clinics, as shown by the infrequent finding of tophi.

Recent prospective epidemiological studies have confirmed the role of lifestyle factors such as excess alcohol, high-purine diet and obesity in the development of gout. ${ }^{17-19}$ To our knowledge, however, only one study has previously examined

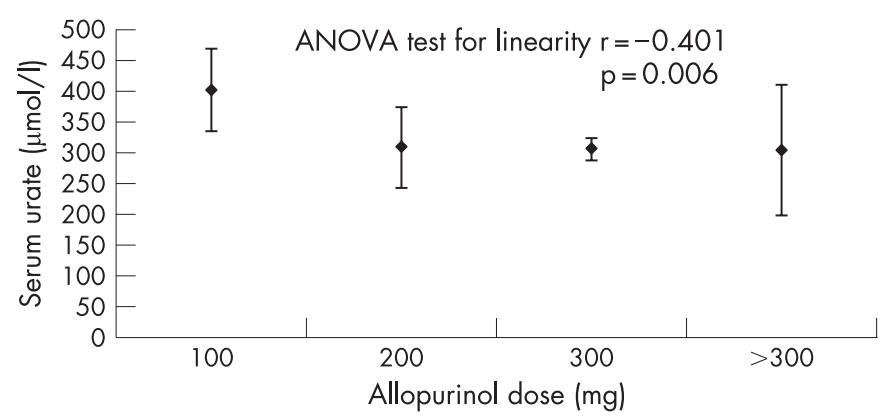

Figure 3 Relationship between serum urate and dose of allopurinol. the frequency with which advice to modify such risk factors is offered to patients with gout. In a review of UK general practice medical records, Pal et al found that advice concerning alcohol and diet was offered to $<30 \%$ of patients. ${ }^{9}$ Our study differs in that we assessed patient recall of lifestyle modification advice, often many years after the event, and although we identified similar results, this approach risks underestimating the frequency of such advice because of poor recall. However, given that patient education is vital for lifestyle modification behaviours to be adopted, it is noteworthy that patient recall of provision of written information and lifestyle modification advice was modest.

Diuretics are widely acknowledged to be a risk factor for hyperuricaemia and subsequently gout, ${ }^{19}$ yet almost twothirds of patients with presumed diuretic-induced gout were still taking a diuretic at the time of the study. Although diuretic cessation should be considered in all patients with gout, this may not be possible in many patients, particularly those with cardiac failure, and an important caveat to the finding that diuretics were frequently continued in the long term is that data regarding the indications for diuretic therapy were not available.

The diagnosis of gout by general practitioners appeared to be reasonably accurate in terms of according with the "specialist" diagnosis based on clinical assessment. The main error encountered was a tendency towards overdiagnosis, rather than underdiagnosis. For such a painful condition, it is perhaps surprising that $12 \%$ of gout sufferers had never consulted a doctor about their gout. Although it is not always easy to differentiate primary from secondary gout, the proportions of those with secondary gout (30\%) and with tophi (6\%) were less than might be expected in hospital-based series, which have a bias towards inclusion of more complex and severe secondary gout cases. It implies, however, that most gout sufferers in the community have the type of gout that is most easily managed and "cured" by standard treatment approaches, making the relative undertreatment in this survey all the more disappointing.

Table 3 Reasons for discontinuation of allopurinol

\begin{tabular}{ll}
\hline Reason & $\mathbf{n}$ \\
\hline Attacks ceased & 9 \\
Patient concern over taking tablets & 3 \\
Rash & 1 \\
Deranged liver-function tests & 1 \\
Patient read that allopurinol can precipitate an acute attack & 1 \\
Temporary measure during an acute attack & 1 \\
Rectal bleeding & 1 \\
Continued attacks & 1 \\
Only taken during chemotherapy (never for gout) & 1 \\
Patient did not know & 2 \\
\hline
\end{tabular}


The major limitation to our study is the low overall response rate to the postal questionnaire. In addition to the 164 gout cases identified, there were a further 89 patients who reported in the questionnaire having gout but did not consent to a faceto-face clinical assessment. Another limitation might be the use of a clinical case definition. Without doubt, the gold standard for diagnosis of gout is identification of crystals on aspirates from synovial fluid or tophus. However, although intercritical joint aspiration is a useful diagnostic technique, ${ }^{20-25}$ the requirement to undergo aspiration might have further reduced the number of people agreeing to participate in the clinical assessment, particularly as only $4 \%$ had experienced joint aspiration previously (data not shown). As an alternative to crystal identification, many studies of gout have used the 1977 ARA preliminary criteria as diagnostic criteria. ${ }^{8}$ However, these classification criteria are limited to the acute arthritis of primary gout, thus they are not helpful for either chronic or secondary gout, and have never undergone further validation as suggested by the authors in the original report. They were included as a secondary diagnostic criterion. The 14 cases of clinically confirmed gout that did not fulfil the 1977 ARA criteria may reflect either cases of secondary gout or misclassification by the clinical approach.

The treatment of chronic gout, a curable form of arthritis, in a primary care-based population appears to be suboptimal and poorly concordant with the recent EULAR recommendations for the management of gout. ${ }^{5}$ The explanation for this observation is likely to be multifactorial. Despite its significant associated comorbidity, gout appears not to be considered an important disease. Previously, doctors have received mixed messages concerning the indications and aims of ULT, the timing of ULT in relation to acute attacks, and the need for and duration of NSAID or colchicine prophylaxis. For example, exactly how many acute attacks of gout constitute "recurrent acute attacks" (proposition 7) indicating a need for ULT remains a subject of much debate. The need to aim for SUA levels well below the saturation threshold of urate (proposition 8) is not widely appreciated. As discussed above, adherence to ULT is thought to be poor. ${ }^{13}{ }^{14}$ There is a need for an education programme to raise the profile of gout and to educate doctors who manage gout about the importance of a multi-faceted treatment strategy and the appropriate use of ULT. Wider dissemination of the EULAR recommendations should be a priority in order to optimise the management of chronic gout.

\section{ACKNOWLEDGEMENTS}

We would like to thank the staff and patients of Arnold Health Centre and The Calverton Practice in Nottingham, UK.

\section{Authors' affiliations \\ Edward Roddy, Weiya Zhang, Michael Doherty, Academic Rheumatology, University of Nottingham, UK}

Funding: We are grateful for funding from the Arthritis Research Campaign, UK (ICAC grant 14851) and unrestricted financial support from Astra-Zeneca-UK and Glaxo-Smith-Kline-USA.

Competing interests: We received an unrestricted grant from Ipsen, France, which contributed towards the cost of the questionnaire survey.

\section{REFERENCES}

1 Mikuls TR, Farrar JT, Bilker WB, Fernandes S, Schumacher HR Jr, Saag KG. Gout epidemiology: results from the UK General Practice Research Database, 19901999. Ann Rheum Dis 2005;64:267-72.

2 Harris CM, Lloyd DC, Lewis J. The prevalence and prophylaxis of gout in England. J Clin Epidemiol 1995:48:1153-8.

3 Lawrence RC, Helmick CG, Arnett FC, Deyo RA, Felson DT, Giannini EH, et al. Estimates of the prevalence of arthritis and selected musculoskeletal disorders in the United States. Arthritis Rheum 1998:41:778-99.

4 Zhang W, Doherty M, Pascual E, Bardin T, Barskova V, Conaghan P, et al. EULAR evidence based recommendations for gout. Part I. Diagnosis: Report of a task force of the Standing Committee for International Clinical Studies Including Therapeutics (ESCISIT). Ann Rheum Dis 2006:65:1301-11.

5 Zhang W, Doherty M, Bardin T, Pascual E, Barskova V, Conaghan P, et al. EULAR evidence based recommendations for gout. Part II. Management: Report of a task force of the Standing Committee for International Clinical Studies Including Therapeutics (ESCISIT). Ann Rheum Dis 2006;65:1312-24.

6 Li-Yu J, Clayburne G, Sieck M, Beutler A, Rull M, Eisner E, et al. Treatment of chronic gout. Can we determine when urate stores are depleted enough to prevent attacks of gout? J Rheumatol $2001 ; 28: 577-80$

7 Shoji A, Yamanaka H, Kamatani N. A retrospective study of the relationship between serum urate level and recurrent attacks of gouty arthritis: evidence for reduction of recurrent gouty arthritis with antihyperuricemic therapy. Arthritis Care Res 2004;51:321-5.

8 Wallace SL, Robinson H, Masi AT, Decker JL, McCarty DJ, Yu TF. Preliminary criteria for the classification of the acute arthritis of primary gout. Arthritis Rheum 1977;20:895-900.

9 Pal B, Foxall M, Dysart T, Carey F, Whittaker M. How is gout managed in primary care? A review of current practice and proposed guidelines. Clin Rheumatol 2000;19:21-5.

10 Devlin JW, Bellamy N, Bayliff CD. Observations and effects of educationa consults on allopurinol prescribing. Can J Hosp Pharm 1992;45:21-7.

11 McClintock AD, Egan AJ, Woods DJ, Pillans PI. A survey of allopurinol dosage prescribing. N Z Med J 1995; 108:346-7.

12 Day RO, Miners JO, Birkett DJ, Whitehead A, Naidoo D, Hayes J, et al. Allopurinol dosage selection: relationships between dose and plasma oxipurinol and urate concentrations and urinary urate excretion. Br J Clin Pharmacol 1988;26:423-8.

13 de Klerk E, van der Heijde D, Landewe R, van der Tempel H, Urquhart J, van der Linden S. Patient compliance in rheumatoid arthritis, polymyalgia rheumatica, and gout. J Rheumatol 2003;30:44-54.

14 Riedel AA, Nelson M, Joseph-Ridge N, Wallace K, MacDonald P, Becker M. Compliance with allopurinol therapy among managed care enrollees with gout: a retrospective analysis of administrative claims. J Rheumatol 2004;31:1575-81.

15 Singer JZ, Wallace SL. The allopurinol hypersensitivity syndrome. Unnecessary morbidity and mortality. Arthritis Rheum 1986;29:82-7.

16 Hande KR, Noone RM, Stone WJ. Severe allopurinol toxicity. Description and guidelines for prevention in patients with renal insufficiency. Am J Med 1984;76:47-56.

17 Choi HK, Atkinson K, Karlson EW, Willett W, Curhan G. Alcohol intake and risk of incident gout in men: a prospective study. Lancet 2004;363:1277-81

18 Choi HK, Atkinson K, Karlson EW, Willett W, Curhan G. Purine-rich foods, dairy and protein intake, and the risk of gout in men. N Engl J Med 2004;350:1093-103.

19 Choi HK, Atkinson K, Karlson EW, Curhan G. Obesity, weight change, hypertension, diuretic use, and risk of gout in men: the health professionals follow-up study. Arch Intern Med 2005; 165:742-8.

20 Agudelo CA, Weinberger A, Schumacher HR, Turner R, Molina J. Definitive diagnosis of gout by identification of urate crystals in asymptomatic metatarsophalangeal joints. Arthritis Rheum 1979;22:559-60.

21 Weinberger A, Schumacher HR, Agudelo CA. Urate crystals in asymptomatic metatarsophalangeal joints. Ann Intern Med 1979;91:56-7.

22 Rouault T, Caldwell DS, Holmes EW. Aspiration of the asymptomatic metatarsophalangeal joint in gout patients and hyperuricemic controls. Arthritis Rheum 1982;25:209-12.

23 Bomalaski JS, Lluberas G, Schumacher HR Jr. Monosodium urate crystals in the knee joints of patients with asymptomatic nontophaceous gout. Arthritis Rheum 1986;29:1480-4.

24 Pascual E. Persistence of monosodium urate crystals and low-grade inflammation in the synovial fluid of patients with untreated gout. Arthritis Rheum 1991;34:141-5.

25 Pascual E, Batlle-Gualda E, Martinez A, Rosas J, Vela P. Synovial fluid analysis for diagnosis of intercritical gout. Ann Intern Med 1999;131:756-9. 